\title{
BMJ Open Shorter or longer anticoagulation to prevent recurrent venous thromboembolism: systematic review and meta-analysis
}

\author{
Luciane Cruz Lopes, ${ }^{1}$ John Eikelboom, ${ }^{2}$ Frederick A Spencer, ${ }^{3}$ Elie A Akl, ${ }^{4,5,6}$ \\ Clive Kearon, ${ }^{2}$ Ignacio Neumann, ${ }^{5,7}$ Sam Schulman, ${ }^{2}$ Neera Bhatnagar, ${ }^{8}$ \\ Gordon Guyatt ${ }^{5}$
}

To cite: Lopes LC, Eikelboom J, Spencer FA, et al. Shorter or longer anticoagulation to prevent recurrent venous thromboembolism: systematic review and metaanalysis. BMJ Open 2014;4: e005674. doi:10.1136/ bmjopen-2014-005674

- Prepublication history and additional material is available. To view please visit the journal (http://dx.doi.org/ 10.1136/bmjopen-2014005674).

Received 17 May 2014 Revised 17 June 2014 Accepted 18 June 2014

CrossMark

For numbered affiliations see end of article.

Correspondence to Dr Luciane Cruz Lopes; luslopes@terra.com.br

\section{ABSTRACT}

Introduction: Venous thromboembolism (VTE) is a major disease associated with short-term and longterm morbidity and mortality. Patients with a VTE provoked by surgery or immobilisation are at low risk of recurrence and do not require long-term anticoagulation; those with a VTE and metastatic cancer are at high risk of recurrence and require lifetime thromboprophylaxis. In those at intermediate risk of recurrence, it remains controversial whether prolonging anticoagulation and thus incurring treatment burden and bleeding risk is warranted.

Methods and analysis: We will conduct a systematic review and meta-analysis of randomised controlled trials enrolling patients with VTE at intermediate risk of recurrence and evaluating short-term anticoagulation (12 weeks to 9 months initial therapy) versus longer term anticoagulation (at least 6 months additional anticoagulation beyond the course of treatment in the shorter arm). Anticoagulation could consist of vitamin $\mathrm{K}$ antagonists or new oral anticoagulants. Outcomes of interest include recurrent non-fatal thrombosis (deep venous thrombosis and pulmonary embolism), major non-fatal bleeding and mortality. We will systematically search CINAHL, EMBASE, MEDLINE and the Cochrane Central Registry of Controlled Trials. Teams of two reviewers will, independently and in duplicate, screen titles and abstracts and complete full text reviews to determine eligibility, and subsequently abstract data and assess risk of bias in eligible trials. We will conduct meta-analyses to establish the effect of shortterm versus long-term anticoagulation on the outcomes of interest and evaluate confidence in estimates (quality of evidence) using the GRADE (grading of recommendations, assessment, development and evaluation) approach.

Ethics and dissemination: Our review will facilitate evidence-based management of patients with unprovoked or recurrent VTE. For purposes of privacy and confidentiality, the systematic review will be limited to studies with deidentified data. The study will be disseminated by peer-review publication and conference presentation.

Trial registration number: PROSPERO (CRD42014007620).

\section{Strengths and limitations of this study}

- The methods of the review are state of the art, and include independent-duplicate study selection, data abstraction and risk of bias assessment.

- We will use of the GRADE approach to assess confidence in estimates of effect.

- We will conduct sensitivity analyses, making progressively more stringent assumptions regarding the outcomes of participants with missing data.

- We will present the results of our meta-analyses in an Evidence Profile to provide a succinct, easily digestible presentation of quality of evidence and magnitude of effects.

- Our results are likely to be limited by limitations in the primary studies.

\section{INTRODUCTION}

Venous thromboembolism (VTE), which comprises deep vein thrombosis (DVT) and pulmonary embolism (PE), is a major disease that results in considerable morbidity and mortality. DVT or PE may occur in almost 2 in 1000 people each year and between $5 \%$ and $15 \%$ of people with untreated DVT may die from PE. ${ }^{1}{ }^{2}$ Thrombosis most commonly affects the deep veins of the lower limbs, but may affect other sites, including the upper limbs. Complications include pulmonary thromboembolism and post-thrombotic syndrome (PTS). ${ }^{3}$

Risk factors for VTE include immobility, surgery (particularly orthopaedic), malignancy, pregnancy, older age, oestrogen therapy and inherited or acquired prothrombotic clotting disorders. ${ }^{4}$ In many patients, DVT remains asymptomatic and resolves without complications. DVTs of concern are those that become symptomatic and are responsible for morbidity and mortality. ${ }^{3}$ Patients with extensive proximal DVT have a substantial risk of developing the PTS, particularly if there is an ipsilateral recurrence with 
further valve destruction. ${ }^{5}$ The average rate of fatal recurrent VTE after anticoagulation is discontinued has been estimated at $0.3 / 100$ patient-years. ${ }^{6}$ On the basis of observational data, ${ }^{78}$ the authors of the ninth iteration of the American College of Chest Physicians (ACCP) antithrombotic guidelines ${ }^{9}$ estimated that in patients with unprovoked proximal DVT or PE the risk of recurrence in the first year after discontinuation of anticoagulation is $10 \%$ with a risk of $5 \%$ per year thereafter (ie, $30 \%$ at 5 years).

A consensus exists regarding the need for anticoagulant treatment, usually with vitamin $\mathrm{K}$ antagonists (VKA) or with novel oral anticoagulants (NOAC) for patients with VTE. Whereas clinicians agree on the need for 36 months of anticoagulation after the diagnosis of VTE, opinions regarding optimal duration of secondary prophylaxis differ. Although the prevention of recurrence is certainly desirable, the risk of major bleeding, together with the burden of therapy and cost, makes long-term treatment potentially problematic.

The risk-to-benefit ratio is highly dependent on the risk of recurrence of VTE, which differs according to the presence or absence of reversible predisposing factors, location of the thrombosis, patient age, presence of comorbid conditions and intrinsic predispositions to thrombosis (inherited and acquired thrombophilia disorders). Guidelines suggest that the risk of recurrence is sufficiently low after a DVT provoked by temporary immobilisation or lower limb fracture and that treatment beyond 6 months is not in the patients' best interests. Further, there is agreement that lifelong anticoagulation is warranted in patients at the highest risk of recurrence (ie, patients with active metastatic cancer). The controversy regarding treatment beyond 6 months is restricted to those with intermediate risk. ${ }^{10} 11$ These patients include those whose VTE was unprovoked or whose VTE, if provoked, had happened more than once (recurrent VTE).

To make optimal decisions, patients and clinicians need best evidence estimates of benefits and harms of shortterm versus long-term anticoagulation. In the trials included in previous systematic reviews of this topic, the anticoagulants administered were VKA. ${ }^{12-15}$ Recent randomised trials have evaluated longer and shorter administration of NOACs. Therefore, we will update a systematic review and meta-analysis of the relative benefits and harms of longer versus shorter periods of anticoagulation in patients at intermediate risk of recurrence. Our primary question will be the impact of indefinite anticoagulation versus discontinuing anticoagulation after 3-9 months on the outcomes of interest. By indefinite anticoagulation we mean continuing anticoagulation until changes in circumstances would mandate a discontinuation. For many such patients, we would anticipate lifetime anticoagulation.

\section{METHODS/DESIGN}

\section{Protocol and registration}

Our protocol is registered on PROSPERO (CRD42014007620), http://www.crd.york.ac.uk/PROSPERO.
Issues in defining trial eligibility

Trials investigating the effect of prolonged anticoagulation on the risk of VTE recurrence vary in terms of the duration of anticoagulation in the shorter and longer duration arms. They also differ in the nature of populations enrolled. These differences create challenges in defining study eligibility criteria.

In defining eligibility criteria, any systematic review faces tension between broad eligibility criteria, which enhance precision of effect estimates and generalisability of results, and narrow criteria, which decrease the risks of heterogeneity and of generating pooled estimates that are not applicable to the range of patients and interventions included. A reasonable strategy for dealing with this tension, which we will adopt, is to choose relatively broad but clinically plausible criteria and then explore possible sources of heterogeneity. Therefore, although standard shorter term anticoagulation is up to 6 months, we are including trials in which the shorter term arm received anticoagulation up to 9 months. For the longer term arm, we will accept any trial in which the duration of treatment is at least 6 months longer than in the shorter term arm. We will conduct subgroup analyses focusing on the duration of therapy in the shorter and longer term arms.

As we described in the background, the controversy regarding duration of anticoagulation is focused on patients in the intermediate risk category. Typically, these are patients with unprovoked VTE or recurrent VTE (provoked and unprovoked), but definitions might differ across trials. Thus, ideally, all patients included in the trials would fall into these risk groups. It would be inappropriate, however, to exclude trials in which most but not all patients fit this description. We will include any study in which, according to the definition used in the study, at least $50 \%$ of patients fall into one of these risk groups. If there is appreciable heterogeneity in the proportion of patients in these risk categories, we will conduct subgroup analyses based on this variability.

\section{Eligibility criteria}

Inclusion

Patients: Studies must include patients with DVT and/or PE in whom at least $50 \%$ have a first unprovoked (no apparent clinical risk factor) ${ }^{16}$ VTE, or a second or subsequent VTE (can be provoked or unprovoked) in the absence of cancer.

Intervention shorter duration treatment: Studies must include an arm in which patients are anticoagulated with either VKA or novel anticoagulants for at least 12 weeks, but no longer than 9 months.

Intervention longer duration of treatment: Studies must include an arm in which patients are anticoagulated with either VKA or novel anticoagulants for at least 6 months longer than in the shorter duration treatment arm.

Outcomes: Trials must report on at least one of the following outcomes: recurrent VTE, DVT, fatal and non- 
fatal pulmonary embolus confirmed by objective testing (for DVT, venography or ultrasonography; for PE radiological imaging including ventilation/perfusion scanning, CT pulmonary angiography, MRI, conventional angiography or autopsy), fatal and non-fatal serious/ important bleeding episodes, PTS, quality of life and total mortality.

Type of study and design: We will include only randomised controlled trials (RCTs), that is, two types of RCT designs. In one design, patients, at the outset of VTE, are randomised to shorter or longer anticoagulation. In the alternative design, all patients undergo the shortcourse anticoagulation regimen. They are then randomised to stop anticoagulation or to a further period of anticoagulation. We will include studies in which patients undergo shorter term treatment with an anticoagulant, most often VKA, and then receive a new anticoagulant versus placebo.

\section{Exclusion}

We will exclude studies enrolling only pure populations of high-risk patients, such as those with protein $\mathrm{S}$ or $\mathrm{C}$ deficiency or antiphospholipid antibody or antiphospholipid antibody syndrome.

\section{Information sources and search}

We will include all RCTs included in the ninth iteration of the ACCP antithrombotic guidelines and then conduct an additional search from January 2011 onwards, 6 months prior (to account for the lag in indexing) to the date of the comprehensive search on the topic for the ninth iteration ACCP antithrombotic guidelines. We will search OVID MEDLINE, EMBASE, the Cochrane Central Registry of Controlled Trials (CENTRAL) and CINAHL with no language restriction. An experienced librarian (NB) developed a sensitive search strategy for this (see online supplementary appendix 1). We will scan the bibliographies of all systematic reviews and meta-analyses as well as all eligible primary studies for additional relevant articles.

\section{Study selection}

Following a calibration exercise, reviewers will work in pairs to screen, independently and in duplicate, titles and available abstracts of identified citations. We will acquire the full text publication of any article that either reviewer judges as potentially eligible. The same reviewer teams will, following a second calibration exercise, independently apply eligibility criteria to the full text of potentially eligible trials using standardised forms. They will resolve disagreements by consensus or, if a discrepancy remains, through discussion with an arbitrator (GG). We will measure inter-rater agreement for full-text eligibility and assessment of risk of bias using the $\kappa$ statistics. Values of $\kappa$ between 0.40 and 0.59 have been considered to reflect fair agreement, between 0.60 and 0.74 to reflect good agreement and 0.75 or more to reflect excellent agreement. ${ }^{17}$
Data collection process and data items

Using pilot tested standardised forms and following a calibration exercise, three teams of two reviewers will extract data independently and in duplicate from each eligible study. Data abstracted will include details on the study methodology, participants, intervention, control and all reported patient-important outcomes. For each outcome, we will record the number of patients enrolled in each study arm, the number of patients for whom final follow-up data are available and the number of events in each study group.

Reviewers will resolve disagreements by discussion, and one arbitrator (GG) will adjudicate unresolved disagreements. We will contact the authors of each study to clarify any issues of uncertainty in the data abstraction and to ensure that our abstraction is correct.

Reviewers will independently extract details of the anticoagulation regimens in the shorter and longer duration arms. Outcomes will include death; cause-specific mortality (PE or bleeding); recurrent non-fatal VTE (DVT and pulmonary embolus) and non-fatal serious/ important bleeding. We will document definitions of serious/important bleeding in each study.

\section{Risk of bias in individual studies}

Reviewers will assess risk of bias within each study with a modified Cochrane risk of bias instrument ${ }^{18}$ which assesses the following key domains: randomisation sequence generation; allocation concealment; blinding of participants, healthcare professionals, outcome assessors, data collectors and data analysts; incomplete outcome data; selective outcome reporting; and other sources of bias which will include differential intensity of surveillance for VTE and bleeding in the short and long arms, and premature cessation of follow-up (for instance, no follow-up for bleeding in the short arm after a VTE and resumption of anticoagulation). We will consider as a criterion of risk of bias whether the investigators specify a total surveillance period equal in length for the longer and shorter arms (ie, same follow-up period from the time of randomisation) versus a total surveillance period that is different (eg, same follow-up period from the time of stopping anticoagulation) or not specified. Reviewers will assign response options of 'definitely yes', 'probably yes', 'probably no' and 'definitely no' for each of the domains, with 'definitely yes' and 'probably yes' ultimately being assigned a low risk of bias and 'definitely no' and 'probably no' a high risk of bias. ${ }^{19}$ Reviewers will resolve disagreements by discussion, and one arbitrator (GG) will adjudicate unresolved disagreements.

\section{Meta-analysis and data synthesis}

Data regarding VTE, serious/important bleeding, mortality and person-time at risk will be extracted by two independent reviewers using a standardised form, with 
adjudication by a third reviewer in cases of disagreement. Given our primary interest in short-term versus indefinite anticoagulation, in studies with follow-up after discontinuation of anticoagulation in the long arm, we will try to identify events that occurred in either arm after the scheduled discontinuation of anticoagulation in the long arm. We will exclude such events in the primary analysis.

For each study, incidence rates in events per personyear at risk will be calculated for the outcomes of recurrent VTE and serious/important bleeding. We have chosen events per person-years rather than number of people with events to account for the differential length of follow-up within individual studies and across studies, and the possibility of multiple events in a single individual. When investigators do not report person-time data (either directly or indirectly through a Kaplan-Meier survival curve), the person-time of the interval will be estimated by multiplying the number of participants present at the beginning of the interval by the duration of the interval and subtracting person-time for events occurring within the interval. For this calculation, we will assume that events will be equally likely throughout the interval unless data to the contrary are in the report.

Our estimates of study heterogeneity will be informed using the $p$ value for $\chi^{2}$ for heterogeneity, and the $\mathrm{I}^{2}$ statistic where $0-40 \%$ may be unimportant heterogeneity, 30-60\% moderate, 50-90\% substantial and 75-100\% considerable heterogeneity (http://www.ncbi.nlm.nih. gov/pmc/articles/PMC192859/). We will explore heterogeneity by conducting the five a priori subgroup analyses using a $\mathrm{z}$-test to test for interaction. ${ }^{20}$

\section{Assessment of heterogeneity and subgroup analyses}

We will explore heterogeneity using subgroup hypotheses, which apply to bleeding and VTE outcomes and mortality and are framed as effects in longer versus shorter duration anticoagulation. We postulate that larger reductions in thrombosis, and larger increases in bleeding, will occur in the following situations: (1) when the shorter duration anticoagulation arm is 3 months or less versus longer than 3 months; (2) when the longer duration anticoagulation arm is more than 12 months longer than the shorter duration arm versus 12 months or less; (3) studies in which the number of risk of bias domains judged as 'high risk' is greater than the median will have larger effects than studies in which that number is less than the median; (4) when therapy is a NOAC versus warfarin with high target international normalised ratio (INR) (lower boundary greater than or equal to 2.0) versus warfarin with lower target INR (lower boundary less than 2.0); and (5) when anticoagulation was continued until the end of the study or in which we can exclude events that occurred in either arm after timing of cessation of anticoagulation in the long arm versus follow-up continued after the anticoagulant stopped and it was not possible to identify and exclude events that occurred after cessation of anticoagulation in the long arm.
Confidence in pooled estimates of effect

Two reviewers will, independently and in duplicate, assess the confidence in effect estimates for each outcome using the GRADE (grading of recommendations, assessment, development and evaluation) rating system. ${ }^{21}$ In the GRADE system of rating quality of evidence for each outcome, randomised trials begin as high-quality evidence, but may be rated down by one or more of five categories of limitations. ${ }^{22}$ The GRADE working group has provided detailed guidance regarding judgements for each of these criteria: (1) risk of bias, ${ }^{23}$ (2) inconsistency, ${ }^{24}$ (3) indirectness, ${ }^{25}$ (4) imprecision $^{26}$ and (5) publication bias. ${ }^{27}$

For assessing the impact of loss to follow-up across studies, we will conduct sensitivity analyses making progressively more stringent assumptions regarding loss to follow-up in intervention and control groups. The extent to which point estimates and CIs differ in these sensitivity analyses will determine whether we should rate down for risk of bias. ${ }^{28}$

We anticipate that the "number of patients enrolled minus number of patients for whom final follow-up is available" may include: (1) unexplained losses to follow-up (lost contact), (2) explained losses to follow-up (eg, followed until patient moved), and (3) Followed until they had a recurrent VTE (or lost to follow-up for other complications such as death, or bleeding from restarted anticoagulation).

With respect to precision, the GRADE guidance notes that meta-analyses of small trials can provide evidence of benefit with CIs that appear to convincingly exclude no effect; however, the results of reviews of such studies have often been subsequently refuted by larger trials. ${ }^{26}$ To address this potential concern in cases in which our meta-analysis suggests benefit but the sample size is less than the optimal information size (OIS: the number of patients generated by a conventional sample size calculation for a single trial), we will rate down the quality for imprecision. For the purposes of calculating the OIS we will assume, for binary variables, a relative risk reduction or increase $(\delta)$ of $25 \%$, an $\alpha$ of 0.05 , a $\beta$ of 0.20 and a median baseline risk from the available studies.

For each outcome, we will assess publication bias by visually observing asymmetry of the funnel plot for each outcome. We will follow published guidance and conduct the funnel plot inquiry only for outcomes with 10 or more trials. ${ }^{27}$

After considering these reasons for rating down, reviewers will judge the overall confidence in estimates of effect for each outcome as follows:

- 'High' quality of evidence (we are very confident that the true effect is close to that of the estimate of the effect);

- 'Moderate' quality of evidence (we are moderately confident in the effect estimate and the true effect is likely to be close to the estimate of the effect, but there is a possibility that it is substantially different); 
- 'Low' quality of evidence (our confidence in the effect estimate is limited and the true effect may be substantially different from the estimate of the effect);

- 'Very low' quality of evidence (we have very little confidence in the effect estimate and the true effect is likely to be substantially different from the estimate of the effect). ${ }^{22}$

Again, we will follow GRADE guidance for overall confidence ratings. ${ }^{29}$

For individual domains and overall confidence, if raters disagree, they will try to resolve by consensus and, if not successful, the final judgement will be made by an independent reviewer (GG).

\section{Presentation of results}

We will present the results of our meta-analyses in an evidence profile that will provide a succinct, easily digestible presentation of quality of evidence and magnitude of effects. ${ }^{30}$ Our evidence profile will be constructed to include the following elements:

1. A measure of the typical burden of these outcomes (eg, control group, estimated risk; if appropriate studies are available, we will use the baseline risk for population-based observational studies);

2. A measure of the difference between the risks with and without intervention;

3. The relative magnitude of effect;

4. Numbers of participants and studies addressing these outcomes;

5. A rating of the overall confidence in estimate of effect for each outcome and any reasons for rating down the confidence.

\section{DISCUSSION}

The decision to continue anticoagulant treatment in patients with VTE at moderate risk of recurrence beyond the first few months is based on the patients' and treating physician's perception of the benefits and harms. The risks of recurrent VTE and bleeding associated with different lengths of VKA treatment have been evaluated in several studies that randomly allocated patients with VTE to receive different lengths of treatment. These studies, subsequently summarised in systematic reviews, were modest in number and of relatively small sample size. Since these studies were completed, new trials have compared shorter and longer durations of treatment with novel anticoagulants. Since the impact of these newer agents on thrombosis and bleeding is similar to that of warfarin, so by including these studies in a new systematic review, we will be able to increase the precision and narrow CIs, thus allowing an improved estimate of effects on thrombosis and bleeding.

Our protocol represents a model for systematic review methods. We have planned standard methods that yield credible results, including explicit eligibility criteria, a comprehensive search, and duplicate assessment of eligibility and risk of bias. We have also planned implementation of methods seldom (a priori hypotheses to explain possible effect modification, including specification of direction of effect) or very seldom (use of the GRADE approach to rating confidence in estimates of effect) implemented in current systematic reviews.

Our review presents several unique challenges. One involves the specification of the study question, and the implications of that specification. Relevant studies used two different designs: short versus longer anticoagulation in which patients in the long arm continued anticoagulation until the end of the study, and short versus fixed longer anticoagulation with continued follow-up after anticoagulation was discontinued. We have decided we are interested in the relative impact of short versus indefinite-rather than fixed longer duration-anticoagulation. This will require exclusion of events in the latter study design that occurred-in the short and long arms-after the end of the longer arm planned anticoagulation. Reported data may limit our ability to carry out this exclusion as would be optimal.

Our study is likely to be limited by other aspects of study design and reporting of the primary studies. In particular, though we are interested in the impact of indefinite versus limited anticoagulation, studies will have limited follow-up, often 2-3 years. Bleeding and event rates in the second and third years will, however, provide a useful estimate of what is liable to happen in subsequent years. Another important limitation is that we will not have access to individual patient data, and therefore subgroup analysis and inferences will be limited.

In conclusion, patients and clinicians choosing between limited and indefinite duration of anticoagulation after VTE deserve access to best estimates of effect derived from the complete current literature. Our review will provide these estimates.

\section{Author affiliations}

${ }^{1}$ Pharmaceutical Sciences Post graduate Course, University of Sorocaba, UNISO, Sorocaba, Sao Paulo, Brazil

${ }^{2}$ Division of Hematology and Thromboembolism, Department of Medicine, McMaster University, Hamilton, Ontario, Canada

${ }^{3}$ Division of Cardiology, Department of Medicine, McMaster University, Hamilton, Ontario, Canada

${ }^{4}$ Department of Internal Medicine, American University of Beirut, Beirut, Lebanon

${ }^{5}$ Department of Clinical Epidemiology and Biostatistics, McMaster University, Hamilton, Ontario, Canada

${ }^{6}$ Department of Medicine, State University of New York at Buffalo, Buffalo, New York, USA

${ }^{7}$ Department of Internal Medicine, School of Medicine, Pontificia Universidad Catolica de Chile, Santiago, Chile

${ }^{8}$ Health Sciences Library McMaster University, Hamilton, Ontario, Canada

Contributors LCL, GG, JE, SS, FAS, CK and EAA conceived the study design. NB and IN designed the database-specific literature search strategies. GG and LCL completed the first draft of the manuscript. All authors reviewed several drafts of the manuscript and approved the final version.

Competing interests JE has taken the position that anticoagulation should not be used long term in the patient population of interest and has tested aspirin in this context. SS is director of an Anticoagulation Clinic. CK led the development of the American College of Chest Physicians antithrombotic 
guidelines addressing anticoagulant use in this population and GG edited the 9th edition of these guidelines. CK is supported by the Jack Hirsh Professorship in Thromboembolism and an Investigator Award from the Heart and Stroke Foundation of Ontario.

\section{Patient consent Obtained.}

Provenance and peer review Not commissioned; externally peer reviewed.

Open Access This is an Open Access article distributed in accordance with the Creative Commons Attribution Non Commercial (CC BY-NC 4.0) license, which permits others to distribute, remix, adapt, build upon this work noncommercially, and license their derivative works on different terms, provided the original work is properly cited and the use is non-commercial. See: http:// creativecommons.org/licenses/by-nc/4.0/

\section{REFERENCES}

1. McManus RJ, Fitzmaurice DA, Murray E, et al. Thromboembolism. Clin Evid 2011;2011:pii:0208.

2. Naess IA, Christiansen SC, Romundstad P, et al. Incidence and mortality of venous thrombosis: a population-based study. $J$ Thromb Haemost 2007;5:692-9.

3. National Guideline C. Prevention and management of venous thromboembolism. A national clinical guideline. Secondary prevention and management of venous thromboembolism. A national clinical guideline. http://www.guideline.gov/content.aspx? id=25639

4. British Thoracic Society Standards of Care Committee Pulmonary Embolism Guideline Development Group. British Thoracic Society guidelines for the management of suspected acute pulmonary embolism. Thorax 2003;58:470-83.

5. Prandoni $P$, Villalta $S$, Bagatella $P$, et al. The clinical course of deep-vein thrombosis. Prospective long-term follow-up of 528 symptomatic patients. Haematologica 1997;82:423-8.

6. Carrier M, Le Gal G, Wells PS, et al. Systematic review: case-fatality rates of recurrent venous thromboembolism and major bleeding events among patients treated for venous thromboembolism. Ann Intern Med 2010;152:578-89.

7. Prandoni $P$, Noventa $F$, Ghirarduzzi $A$, et al. The risk of recurrent venous thromboembolism after discontinuing anticoagulation in patients with acute proximal deep vein thrombosis or pulmonary embolism. A prospective cohort study in 1,626 patients. Haematologica 2007;92:199-205.

8. Rodger MA, Kahn SR, Wells PS, et al. Identifying unprovoked thromboembolism patients at low risk for recurrence who can discontinue anticoagulant therapy. CMAJ 2008;179:417-26.

9. Kearon C, Akl EA, Comerota AJ, et al. Antithrombotic therapy for VTE disease: Antithrombotic Therapy and Prevention of Thrombosis, 9th ed: American College of Chest Physicians Evidence-Based Clinical Practice Guidelines. Chest 2012;141(2 Suppl):e419S-94S

10. Kearon C, lorio A, Palareti G, et al. Risk of recurrent venous thromboembolism after stopping treatment in cohort studies: recommendation for acceptable rates and standardized reporting. Thromb Haemost 2010;8:2313-15.

11. Kearon C. A conceptual framework for two phases of anticoagulant treatment of venous thromboembolism. J Thromb Haemost 2012;10:507-11.
12. East AT, Wakefield TW. What is the optimal duration of treatment for DVT? An update on evidence-based medicine of treatment for DVT. Semin Vasc Surg 2010;23:182-91.

13. Holbrook A, Schulman S, Witt DM, et al. Evidence-based management of anticoagulant therapy: antithrombotic therapy and prevention of thrombosis, 9th edn: American College of Chest Physicians Evidence-Based Clinical Practice Guidelines. Chest 2012;141(2 Suppl):e152S-84S.

14. Boutitie F, Pinede L, Schulman S, et al. Influence of preceding length of anticoagulant treatment and initial presentation of venous thromboembolism on risk of recurrence after stopping treatment: analysis of individual participants' data from seven trials. BMJ 2011;342:d3036.

15. van Dongen CJ, Vink R, Hutten BA, et al. The incidence of recurrent venous thromboembolism after treatment with vitamin $\mathrm{K}$ antagonists in relation to time since first event: a meta-analysis. Arch Intern Med 2003:163:1285-93.

16. Kearon C, Kahn SR, Agnelli G, et al. Antithrombotic therapy for venous thromboembolic disease: American College of Chest Physicians Evidence-Based Clinical Practice Guidelines (8th Edition). Chest 2008;133(6 Suppl):454S-545S.

17. Orwin RG. Evaluating coding decisions. In: Cooper H, Hedges LV. eds. The handbook of research synthesis. New York, NY: Russell Sage Foundation, 1994:139-62.

18. Busse JW, Guyatt G. Modification of Cochrane tool to assess risk of bias in randomized trials. 2013. http://distillercercom/resources/

19. Akl EA, Sun X, Busse JW, et al. Specific instructions for estimating unclearly reported blinding status in randomized trials were reliable and valid. J Clin Epidemiol 2012;65:262-7.

20. Altman DG, Bland JM. Interaction revisited: the difference between two estimates. BMJ 2003;326:219.

21. Atkins $D$, Best $D$, Briss PA, et al. Grading quality of evidence and strength of recommendations. BMJ 2004;328:1490.

22. Guyatt GH, Oxman AD, Vist GE, et al. GRADE: an emerging consensus on rating quality of evidence and strength of recommendations. BMJ 2008;336:924-6.

23. Guyatt GH, Oxman AD, Vist G, et al. GRADE guidelines: 4. Rating the quality of evidence-study limitations (risk of bias). J Clin Epidemiol 2011;64:407-15.

24. Guyatt GH, Oxman AD, Kunz R, et al. GRADE guidelines: 7. Rating the quality of evidence-inconsistency. J Clin Epidemiol 2011;64:1294-302.

25. Guyatt GH, Oxman AD, Kunz R, et al. GRADE guidelines: 8. Rating the quality of evidence -indirectness. J Clin Epidemiol 2011:64:1303-10.

26. Guyatt GH, Oxman AD, Kunz R, et al. GRADE guidelines 6. Rating the quality of evidence-imprecision. $J$ Clin Epidemiol 2011;64:1283-93.

27. Guyatt GH, Oxman AD, Montori V, et al. GRADE guidelines: 5 . Rating the quality of evidence-publication bias. $J$ Clin Epidemiol 2011;64:1277-82.

28. Akl EA, Johnston BC, Alonso-Coello P, et al. Addressing dichotomous data for participants excluded from trial analysis: a guide for systematic reviewers. PLOS ONE 2013;8:e57132.

29. Guyatt G, Oxman AD, Sultan $S$, et al. GRADE guidelines: 11 Making an overall rating of confidence in effect estimates for a single outcome and for all outcomes. J Clin Epidemiol 2013;66:151-7.

30. Guyatt GH, Oxman AD, Santesso N, et al. GRADE guidelines: 12. Preparing summary of findings tables-binary outcomes. $J$ Clin Epidemiol 2013;66:158-72. 